\title{
miR-223 functions as a potent tumor suppressor of the Lewis lung carcinoma cell line by targeting insulin-like growth factor-1 receptor and cyclin-dependent kinase 2
}

\author{
WEIQI NIAN ${ }^{1,2}, \mathrm{XUJUN} \mathrm{AO}^{2,3}$, YONGZHONG WU $^{1}$, YI HUANG $^{4}$, JIANGHE SHAO ${ }^{1}$, \\ YIMING WANG $^{1}$, ZHENGTANG CHEN ${ }^{2}$, FANGLIN CHEN ${ }^{2}$ and DONGLIN WANG ${ }^{1}$ \\ ${ }^{1}$ Department of Oncology, Chongqing Tumor Hospital, Chongqing 400030; ${ }^{2}$ Institute of Cancer Research, \\ Xinqiao Hospital, Third Military Medical University, Chongqing 400037; ${ }^{3}$ Department of General Surgery, \\ 532 Hospital of the People's Liberation Army, Huangshan, Anhui $245041 ;{ }^{4}$ Center for Clinical Molecular Medicine, \\ Children's Hospital, Chongqing Medical University, Chongqing 400014, P.R. China
}

Received December 19, 2012; Accepted May 14, 2013

DOI: $10.3892 / 01.2013 .1375$

\begin{abstract}
RNAs (miRNAs) have been hypothesized to function as oncogenes or tumor suppressors by targeting specific cancer-related genes. Previous studies have reported that miR-223 may serve as a tumor suppressor in a number of cancer types, however, knowledge of its targets in non-small cell lung cancer (NSCLC) remains limited. In the current study, miR-223 was found to inhibit cell proliferation in vitro by CCK-8 assay, growth curves and an anchorage-independent growth assay in a Lewis lung carcinoma (LLC) cell line. miR-223 transfection in the LLC cells was observed to significantly inhibit migration and invasion, induce $G_{2} / M$ arrest and decrease the expression levels of Sca-1, a marker of murine stem cells. In addition, miR-223 transfection markedly suppressed AKT and ERK signaling, as well as insulin-like growth factor-1 receptor (IGF-1R)-mediated downstream signaling, pathways that are crucial for cell proliferation and invasion in NSCLC cells. Analyses in C57BL/6 mice demonstrated that miR-223 suppresses tumorigenicity in vivo. Using a luciferase activity assay and western blot analysis, IGF-1R and cyclin-dependent kinase 2
\end{abstract}

Correspondence to: Dr Donglin Wang, Department of Oncology, Chongqing Tumor Hospital, 181 Hanyu Road, Chongqing 400030, P.R. China

E-mail: wangdonglincqth@126.com

Dr Fanglin Chen, Institute of Cancer Research, Xinqiao Hospital, Third Military Medical University, 183 Xinqiao Street, Chongqing 400037, P.R. China

E-mail: chenfanglin77@yahoo.com.cn

*Contributed equally

Key words: non-small cell lung cancer, miR-223, tumor suppressor, metastasis, self-renewal
(CDK2) were identified as direct targets of miR-223. In the present study, novel cancer-related targets of miR-223 were identified and verified in a LLC cell line, indicating that miR-223 functions as a tumor suppressor, which may fine-tune the activity of the IGF-1R pathway in lung cancer. Therefore, increasing miR-223 expression may provide a novel approach for the treatment of NSCLC.

\section{Introduction}

Lung cancer is the most common cancer worldwide in terms of incidence and mortality, and its incidence is rapidly increasing in developing countries (1-2). The prognosis of lung cancer remains poor despite recent advances in chemotherapies and molecular-targeted therapies. The five-year survival rate of lung cancer is $<15 \%$, and $\sim 90 \%$ of mortalities are caused by metastasis. To improve patient survival, the elucidation of the regulatory mechanisms that control the tumor metastatic properties of lung cancer is urgently required.

microRNAs (miRNAs) are small non-coding RNA molecules that suppress gene expression by interacting with the $3^{\prime}$ untranslated regions (UTRs) of target mRNAs (3). Although miRNAs account for only a minor fraction of the expressed genome, these RNAs are involved in modulating a number of cellular pathways, including proliferation (4), differentiation (5) and apoptosis (6). The deletion or epigenetic silencing of miRNAs that normally repress the expression of one or more oncogenes may lead to carcinogenesis (7). Thus, miRNAs have been hypothesized to function as tumor suppressors or oncogenes, and alterations in miRNA expression may be critical for tumorigenesis and cancer progression (8-10). miR-223 is a highly conserved miRNA and is crucial for triggering the myeloid differentiation of progenitor cells (11) and for maintaining granulocyte function (12). Previous studies have reported a number of significant miR-223 targets associated with malignancy, including insulin-like growth factor-1 receptor (IGF-1R) (13), myocyte enhancer factor 2C (14), stathmin 1 (15), artemin (16) and FOXO1A (17). 
miR-223 has been reported to be markedly downregulated in the lungs of rats exposed to environmental cigarette smoke for 28 days (18). The reduced serum expression of miR-223 was found to be associated with cancer-specific mortality in stage IA/B patients (19). In our previous study, CXCR4-positive cells from the Lewis lung carcinoma (LLC) cell line presented with cancer metastatic stem cell characteristics, and miR-223 expression was reduced compared with in CXCR4-negative cells $(20,21)$. However, the mechanism by which miR-223 functions in the development of NSCLC remains largely unknown and, to date, no miR-223 targets have been reported in NSCLC. Therefore, in the present study, the effects of miR-223 transfection in the LLC cell line were evaluated to determine whether miR-223 functions as a tumor suppressor of lung cancer.

\section{Materials and methods}

Cell line and culture. The LLC cell line was purchased from the American Type Culture Collection (Manassas, VA, USA). The LLC cells were cultured in Dulbecco's modified Eagle's medium (DMEM) with $10 \%$ fetal calf serum, $100 \mathrm{U} / \mathrm{ml}$ streptomycin and $100 \mathrm{U} / \mathrm{ml}$ penicillin in a humidified atmosphere of $95 \%$ air and $5 \% \mathrm{CO}_{2}$ at $37^{\circ} \mathrm{C}$.

Cell transfection. For the functional analysis, miR-223 and non-targeting miRNA mimics (Thermo Fisher Scientific, Inc., Waltham, MA, USA) were used. For the target validation and cell signaling analysis, the mmu-miRNA-223 expression vector and an empty pGenesil-1.1 (Wuhan Genesil Biotechnology Co Ltd., Hubei, China) were used. The mimics were transfected into the appropriate cells using Lipofectamine 2000 (Invitrogen Life Technologies, Carlsbad, CA, USA) according to the manufacturer's instructions.

Cell survival assays. The effects of miRNA-223 expression on LLC proliferation were assessed using Cell Counting Kit- 8 (CCK-8; Dojindo Laboratories, Kumamoto, Japan). Briefly, the cells were plated on 96-well plates. Following transfection, CCK-8 was added to each well at various times and incubated at $37^{\circ} \mathrm{C}$ for $1.5 \mathrm{~h}$. The absorbance at $450 \mathrm{nM}$ was measured using a microplate spectrophotometer (Tecan Group Ltd, Männedorf, Switzerland).

Anchorage-independent growth ability assay. In total, 500 cells were trypsinized and suspended in $2 \mathrm{ml}$ complete medium with $0.3 \%$ agar (Sigma-Aldrich, St Louis, MO, USA). The agar-cell mixture was plated on top of a bottom layer with $1 \%$ complete medium agar mixture. Subsequent to 10 days, the viable colonies containing $>50$ cells or those $>0.1 \mathrm{~mm}$ in size were counted. The colony size was measured using an ocular micrometer.

Invasion assay. The LLC measurements were performed in 24-well matrigel-coated invasion chambers. The lower chambers contained $600 \mu \mathrm{l}$ DMEM with $10 \%$ fetal bovine serum (FBS) as a chemoattractant. At $24 \mathrm{~h}$ post-transfection with miRNA-223 or non-targeting miRNA mimics, a cell suspension of $5 \times 10^{4}$ cells in $100 \mu \mathrm{l}$ DMEM with $0.5 \%$ FBS was added to the upper chamber. Following incubation for
$24 \mathrm{~h}$ at $37^{\circ} \mathrm{C}$ in a humidified incubator with $5 \% \mathrm{CO}_{2}$, the invasive cells that had attached to the lower surface of the membrane insert were fixed by $4 \%$ formaldehyde and stained with crystal violet. The number of cells was then quantified under a microscope.

Wound healing assay. For the wound healing assay, the LLC cells were grown to confluence on 6-well plates. Next, linear scratch wounds were created on the confluent monolayer using a pipette tip and the cells were transfected with $50 \mathrm{nM}$ miR-223 mimics or $50 \mathrm{nM}$ non-targeting miRNA mimics (control). Floating cells were removed by gentle washes with culture medium. The healing process was examined dynamically and was recorded with a digital camera $24 \mathrm{~h}$ after wound generation.

Matrix metallopeptidase (MMP)9 enzyme-linked immunosorbent assay (ELISA). Following transfection with miRNA-223 or non-targeting miRNA mimics, MMP9 protein levels in the culture supernatants were quantified using an ELISA kit according to the manufacturer's instructions (R\&D Systems, Minneapolis, MN, USA). Plates were read at $450 \mathrm{nM}$ using a Synergy HT microplate reader (BioTek Instruments, Inc., Winooski, VT, USA) and the MMP9 concentration in the samples was calculated using a standard curve.

Flow cytometric analysis. At 48 h post-transfection with miRNA-223 or non-targeting miRNA mimics, dissociated LLC cells were stained with PE-conjugated (PE) rat anti-mouse Sca-1 and the corresponding isotype controls (1:100; BD Pharmingen, San Diago, CA, USA). Briefly, the cells were trypsinized; the trypsin was neutralized with culture medium containing adult bovine serum (ABS) and centrifuged at $450 \mathrm{x} \mathrm{g}$ for $5 \mathrm{~min}$. The cell pellet was re-suspended in Hank's buffered salt solution (HBSS) containing 2\% ABS $\left(\mathrm{HBSS}^{+}\right)$. The cells were incubated with primary antibodies for $20 \mathrm{~min}$ on ice, washed twice with $\mathrm{HBSS}^{+}$and re-suspended with $\mathrm{HBSS}^{+}$containing Sytox Blue or Sytox Red (Invitrogen Life Technologies) to exclude any dead cells. The cell suspensions were filtered using 40- $\mu \mathrm{m}$ filters and analyzed on a BD LSRII Fortessa (both BD Biosciences, Franklin Lakes, NJ, USA). In addition, a cell cycle analysis was performed as described previously (22).

In vivo tumorigenicity. Female C57BL/6 mice (6-8 weeks old) were obtained from The Animal Facility of The Third Military Medical University (Chongqing, China). In vivo experiments were performed in accordance with institutional guidelines. This study was approved by the ethics committee of Chongqing Tumor Hospital, Chongqing, China. Two groups of mice were tested. Group 1 (223-mimic) was injected with LLC cells transfected with miR-223 mimics and group 2 (mimic-control) was injected with LLC cells transfected with non-targeting miRNA mimics. The mice were sacrificed at four weeks, and the tumor volume was calculated using the following formula: $\left[\mathrm{L} \times(\mathrm{W})^{2}\right] / 2$, where $\mathrm{L}$ is the length and $\mathrm{W}$ is the width of the tumor. For the immunofluorescence labeling, the cells were blocked with $15 \%$ bovine serum albumin (BSA) for $1 \mathrm{~h}$ and then incubated at $37^{\circ} \mathrm{C}$ for $1 \mathrm{~h}$ in PE-conjugated rat anti-mouse Sca-1 (1:100). Following this, 
A

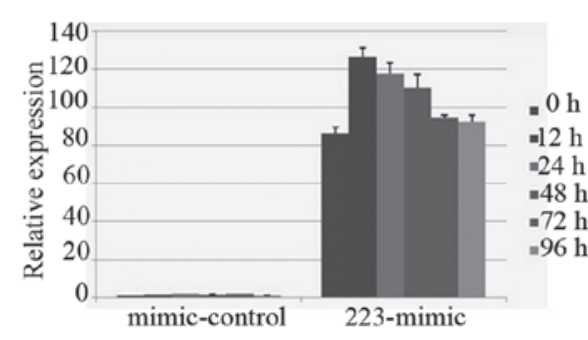

C

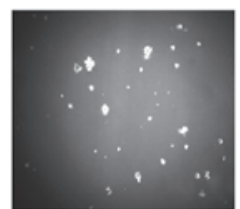

mimic-control

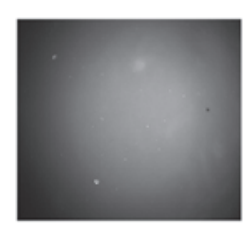

223-mimic
B
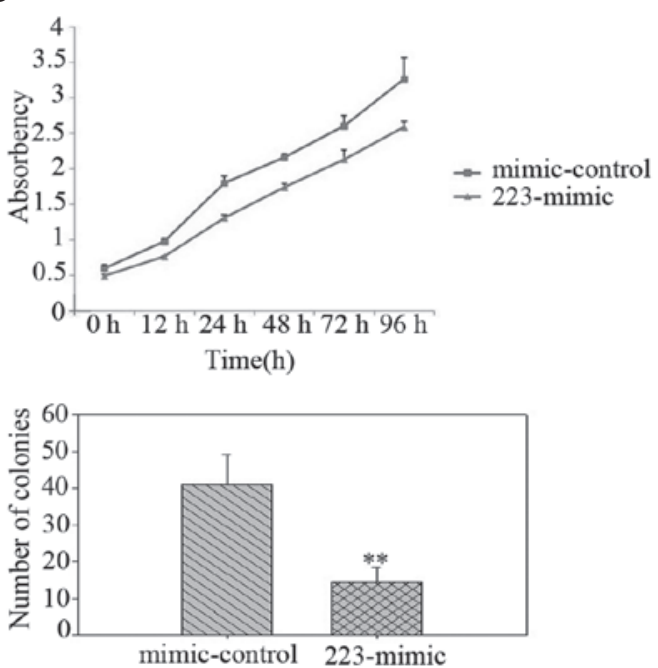

Figure 1. miR-223 overexpression reduces proliferation and clonogenic cell growth. (A) Effect of miR-223-mimic transfection into LLC cells was confirmed by quantitative (q)PCR. (B) Growth curves of miR-223- and control mimic-transfected LLC cells were conducted by CCK-8 assay. (C) Upregulation of miR-223 inhibited LLC tumorigenicity as determined by anchorage-independent growth assay. Magnification, $\mathrm{x} 100$. Data are presented as the mean \pm SD of three replicates. ${ }^{*} * \mathrm{P}<0.01$ vs. mimic-control. miR, microRNA; LLC, Lewis lung carcinoma; CCK-8, Cell Counting kit-8.

the cells were washed with $0.01 \%$ PBS and then stained with 4,6-diamidino-2-phenylindole (Sigma-Aldrich) to identify the cell nuclei. The cells were then observed under a fluorescence microscope.

Luciferase reporter plasmid construction and luciferase assay. The pMIR-REPORT miRNA expression reporter (firefly luciferase reporter plasmid; Life Technologies, Grand Island, NY, USA) was used for the plasmid construction. Constructs were generated using the following primers: IGF-1R 3'-UTR-1, 5'-GGACTAGTAGGGGAGAGCAGGTTG TAACAATCT-3' and 5'-CGACGCGTGACCTACGGTGTC AGGCAGGTGTAT-3'; IGF-1R 3'-UTR-2, 5'-GGACTAGTC AGTACCTGACAGTAGGCCAATGAT-3' and 5'-CGACGC GTAAGATTTGGTCAGTCCTTGTTTAGC-3'; cyclin-dependent kinase (CDK)2 3'-UTR: 5'-GGACTAGTAGCCTTCTGA TGTTTTCTGGCTGTC-3' and 5'-CGACGCGTGATGAAC AGACCAGAGTGACGTGCA-3'. The 3'-UTR and miR-223 complementary sequence (TGGGGTATTTGACAAACT GACA) were separately cloned into the pMIR-REPORT plasmid (Life Technologies), according to the manufacturer's instructions. Constructs ( $0.05 \mu \mathrm{g}$ each) were cotransfected into 293T with $0.01 \mu \mathrm{g}$ a Renilla luciferase control vector using calcium phosphate transfection. Luciferase activity was measured $36 \mathrm{~h}$ after transfection and normalized against Renilla activity, according to the manufacturer's instructions (Dual-Luciferase Reporter Assay System; Promega Corporation, Madison, WI, USA).

Quantitative $(q) P C R$. To determine the gene expression levels, qPCR was performed using the Quantitect SYBR PCR kit (Qiagen, Hilden, Germany), according to the manufacturer's instructions. The primers selected were as follows: IGF-1R forward, 5'-AAGCCGATGTGTGAGAAGACC-3' and reverse, 5'-ATAGTAGTAGTAGTG GCGGCAAGC-3'; CDK2 forward, 5'-TTCATGGATGCCTCTGCTCTC-3' and reverse, 5'-TCC AAAAGCTCTGGCTAGTCC-3'; MMP9 forward, 5'-GTG GAGAGTCGAAATCTCTGG-3' and reverse, 5'-TTTGGA ATCTGCCCAGGTCTG-3'; GAPDH forward, 5'-TGGTAT CGTGGAAGGACTCATGAC-3' and reverse, 5'-ATGCCA GTGAGCTTCCCGTTCAGC-3'. $\Delta \Delta \mathrm{C}_{\mathrm{T}}$ values were normalized against those obtained from the amplification of GAPDH. All reactions were performed in triplicate.

Western blot analysis. Transfected cells in culture were harvested at various times, washed once with cold PBS and lysed in buffer containing protease inhibitors. The protein concentrations from whole cultured cells were measured with the bicinchoninic acid protein assay kit (Beyotime Institute of Biotechnology, Shanghai, China), using BSA as the standard. Protein $(30 \mu \mathrm{g})$ was separated by SDS-PAGE using a $10 \%$ polyacrylamide gel and then electroblotted onto a nitrocellulose membrane. The membrane was immunoblotted overnight at $4^{\circ} \mathrm{C}$ with the primary antibodies. The following antibodies were used at a 1:1,000 dilution: anti-IGF-IR, anti-mmp9 (Santa Cruz Technologies, Santa Cruz, CA, USA), anti-phospho-IGFIR, anti-phospho-Akt, anti-Akt, anti-p44/42 MAPK (Erk1/2), anti-phospho-Erk1/2 and anti-CDK2 (Cell Signaling Technology, Beverly, MA, USA). A goat anti-rabbit horseradish peroxiadse (HRP) secondary antibody (Wuhan Boster Bio-Engineering Co, Ltd, Wuhan, China) was used at a 1:2,000 dilution. The proteins were detected using an enhanced chemiluminescence kit (Pierce Biotechnology, Inc., Rockford, IL, USA), according to the manufacturer's instructions. GAPDH was used as an internal control.

Statistical analysis. Data are presented as the mean \pm SD and analyzed using SPSS 16.0 (SPSS, Inc., Chicago, IL, USA). To assess the statistical significance of the differences, an unpaired t-test was performed. $\mathrm{P}<0.05$ was considered to indicate a statistically significant difference. 


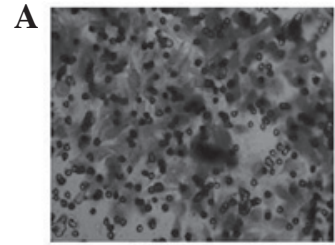

mimic-control

B
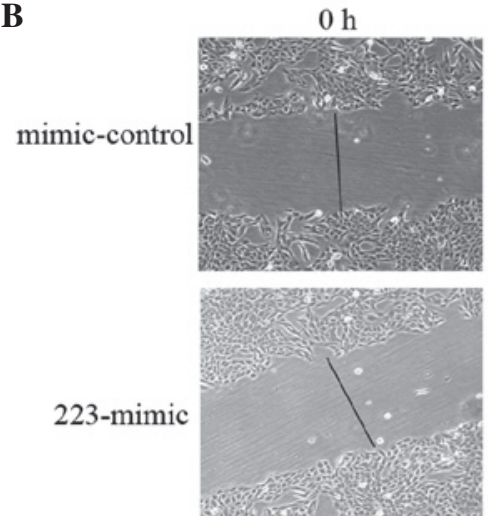

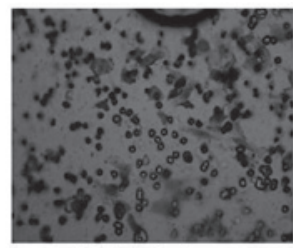

223-mimic

$24 \mathrm{~h}$
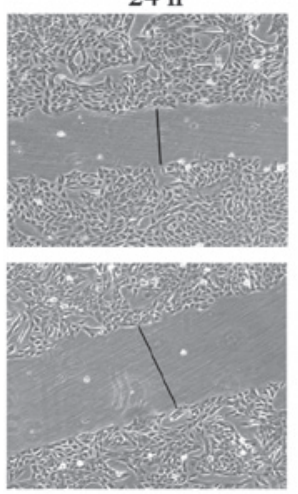

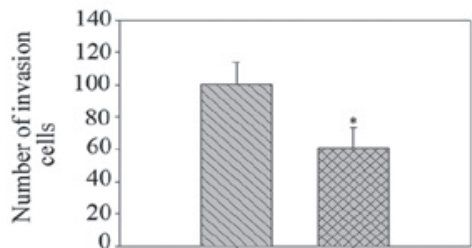

mimic-control 223-mimic

$\mathbf{C}$

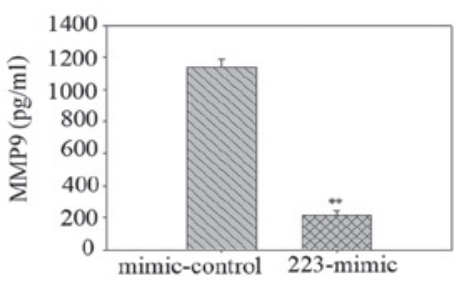

Figure 2. miR-223 overexpression inhibits invasion in LLC cells. (A) Invasion assay for miR-223- and control mimic-transfected cells. Magnifcation, x200. Relative quantification of invaded cells from transwell invasion assay are presented. (B) Representative images of wound healing ( $24 \mathrm{~h}$ after scratch) in miR-223 and control mimic-transfected cells are presented. Magnifcation, x200. (C) At $48 \mathrm{~h}$ post-transfection, expression of MMP-9 protein in culture supernatants was estimated by enzyme-linked immunosorbent assay (ELISA). ${ }^{*} \mathrm{P}<0.05$ and ${ }^{* *} \mathrm{P}<0.01$, vs. mimic-control. miR, microRNA; LLC, Lewis lung carcinoma.

\section{Results}

Upregulation of miRNA-223 suppresses proliferation and tumorigenicity of LLC cells. To investigate the biological role of miR-223 expression in the development and progression of lung cancer, LLC cells were transfected with miR-223 mimics and the effect on cellular proliferation was assessed. Following transfection, the miR-223 levels were increased in the LLC cells, indicating that the increase was due to miR-223 transfection (Fig. 1A). Using a CCK-8 assay, the overexpression of miR-223 (223-mimic) was observed to markedly reduce the growth rate of the LLC cells compared with that of the non-targeting miRNA mimic-transfected cells (mimic-control; Fig. 1B). Notably, the LLC cells ectopically expressing miR-223 were identified to exhibit a significantly inhibited anchorage-independent growth ability, as demonstrated by the decrease in colony numbers and sizes (Fig. 1C), indicating that upregulation of miR-223 reduces the tumorigenicity of lung cancer cells in vitro.

Ectopic expression of miRNA-223 inhibits LLC invasion. To examine invasion, the LLC cells were transfected with miR-223 or control mimics and reseeded on top of the insert. Subsequent to $48 \mathrm{~h}$, the number of transmembrane cells in the 223 -mimic group $(60.67 \pm 12.66)$ was lower than that of the mimic-control group (100.33 \pm 14.01 ; P $<0.05$; Fig. 2A). Next, the LLC cells were transfected as described, scratch wounds were generated and cell migration towards the wound was visualised. The wound healing assay revealed that miR-223 reduced the motility of the LLC cells (Fig. 2B). To determine whether the increased invasion observed was correlated with concomitant changes in MMP levels, the total active MMP9 protein levels were measured by ELISA in the cultured media.
The MMP9 levels in the supernatant of miR-223-overexpressing cells $(214.16 \pm 28.18 \mathrm{pg} / \mathrm{ml})$ were reduced compared with that of the mimic-controls $(1,139.14 \pm 50.13 \mathrm{pg} / \mathrm{ml} ; \mathrm{P}<0.01$; Fig. 2C). These observations indicated that miR-223 inhibits invasion in LLC cells.

Overexpression of miR-223 in LLC cells induces $G_{2} / M$ phase arrest and reduces Sca-1 protein expression. Propidium iodide staining of miR-223-overexpressing LLC cells revealed an increase in the $\mathrm{G}_{2} / \mathrm{M}$ cell populations $(13.3 \pm 0.85$ vs. $.30 \pm 0.46 \%)$ and a decrease in cells in the $\mathrm{G}_{0} / \mathrm{G}_{1}$ phase populations $(45.00 \pm 1.11$ vs. $54.46 \pm 0.85 \%)$ compared with the mimic-control $(\mathrm{P}<0.01$; Fig. 3A), indicating a block in the $\mathrm{G}_{2} / \mathrm{M}$ phase transition of the cell cycle. Notably, the percentage of Sca-1-positive cells (Sca-1 is a well-known marker in murine stem cells) was reduced from $39.25 \pm 2.36$ to $17.47 \pm 2.70 \%$ in the miR-223-overexpressing group $(\mathrm{P}<0.01$; Fig. 3B).

miR-223 expression in LLC inhibits tumor growth in vivo. At four weeks post-injection, the mice administered with miR-223 mimics had formed markedly smaller tumors than the mimic-control group (Fig. 4A). The tumor volume subsequent to sacrifice in mice injected with miR-223 mimic-transfected cells was $2,034.30 \pm 983.99 \mathrm{~mm}^{3}$, whereas the tumor volume in mice injected with the control mimic-transfected cells was $5,860.20 \pm 692.58 \mathrm{~mm}^{3}$. The Sca-1 expression in the tumor tissue from the miR-223 mimic-transfected cells was significantly lower than that of the control mimic-transfected cells, as demonstrated by immunofluorescence staining (Fig. 4B).

miR-223 regulates $I G F-1 R$ and $C D K 2$ levels by binding to the $3^{\prime} U T R$. Bioinformatic analyses revealed that the IGF-1R 

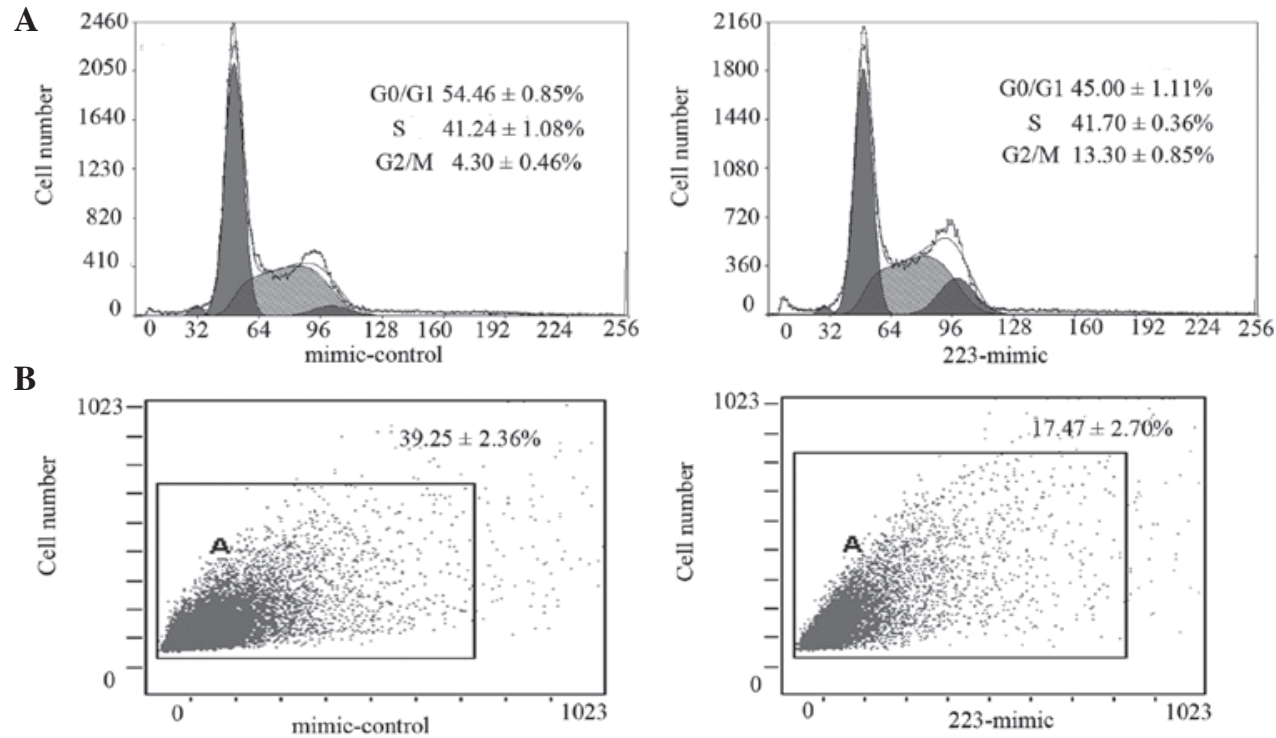

Figure 3. miR-223 affects the cell cycle and self renewal in LLC cells. (A) Fluorescence-activated cell sorting (FACS) analysis of the cell cycle status of 223-mimic and mimic-control transfected cells. (B) FACS analysis of the Sca-1 expression in 223-mimic and mimic-control transfected cells. Data are presented as the mean $\pm \mathrm{SD}$ of three replicates. miR, microRNA; LLC, Lewis lung carcinoma.

A

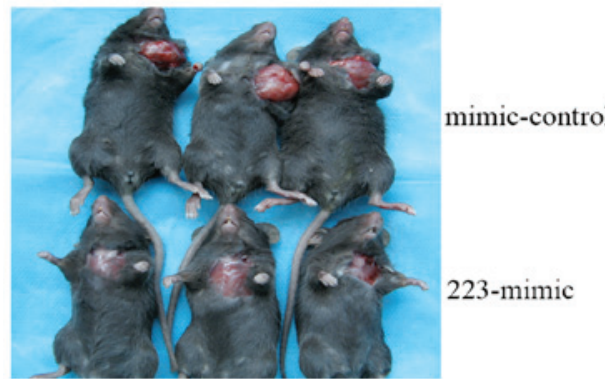

B
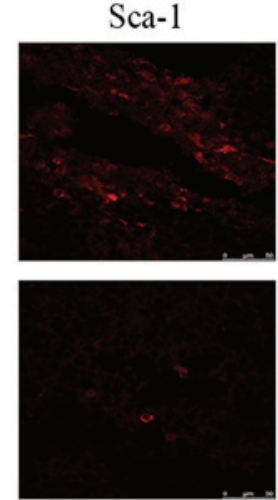

DAPI
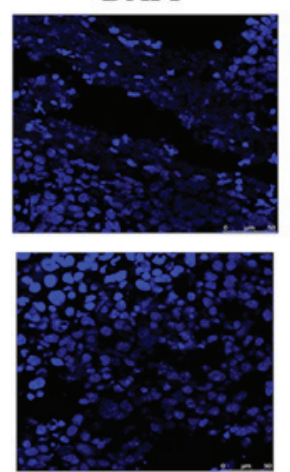

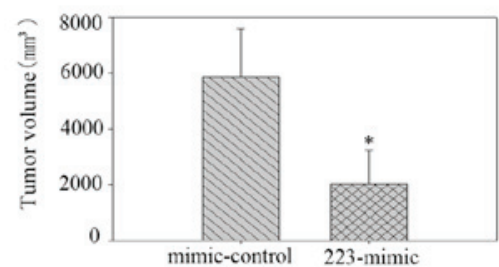

merge

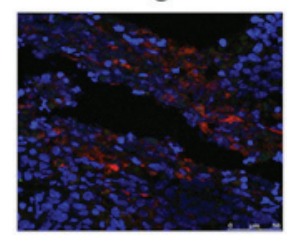

mimic-control

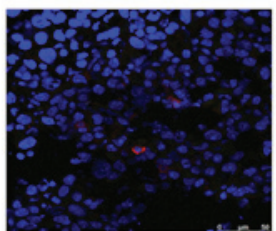

Figure 4. In vivo functional studies on the effect of miR-223-treatment on LLC cells in C57BL/6 mice. (A) Decreasing trend in size of tumors from miR-223to control mimic-transfected cells. "P<0.05 vs. mimic-control. (B) Sca-1 expression was analyzed by fluorescence microscopy of LLC tissues treated with miR-223 and control mimics. Cell nuclei were stained with 4,6-diamidino-2-phenylindole (magnifcation, x100). miR, microRNA; LLC, Lewis lung carcinoma.

3'UTR contained two putative miR-223 binding sites for miR-223 and CDK2 contained one putative miR-223 binding site for miR-223 (Fig. 5A). The luciferase activities of the IGF-1R 3'UTR, CDK2 3'UTR and miR-223 complementary sequence-containing constructs were found to be further repressed in cells overexpressing miR-223 (Fig. 5B). Next, to examine whether miR-223 affects IGF-1R and CDK2 expression in LLC, the mRNA and protein expression levels of IGF-1R and CDK2 were analyzed using qPCR and western blot analysis. Subsequent to $48 \mathrm{~h}$, the expression of IGF-1R and
CDK2 mRNA in the miR-223-expressing group was reduced by $\sim 17$ - and 25 -fold of the control vector group, respectively (Fig. 5C). miR-223 also caused a significant reduction in the IGF-1R and CDK2 protein levels (Fig. 5D). These results indicate that IGF-1R and CDK-2 are post-transcriptionally regulated by miR-223 in LLC cells.

Two pathways have been described for IGF-IR, the phosphatidylinositol-3 kinase-Akt and mitogen-activated protein kinase pathways (also known as ERKs) (23). To determine the consequences of the interference of IGF-1R expression 


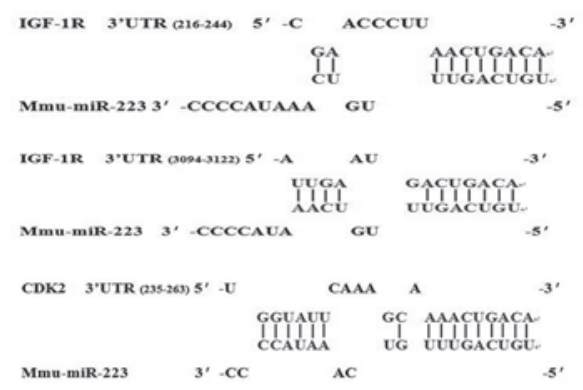

C

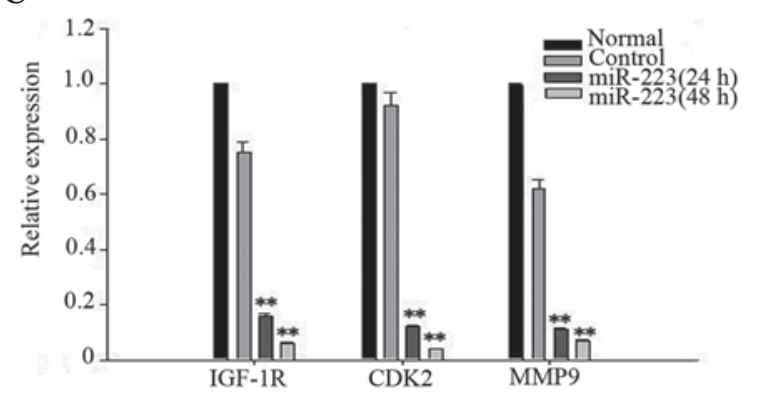

B

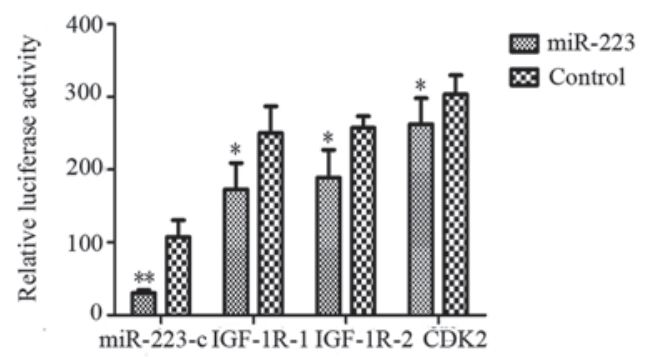

D

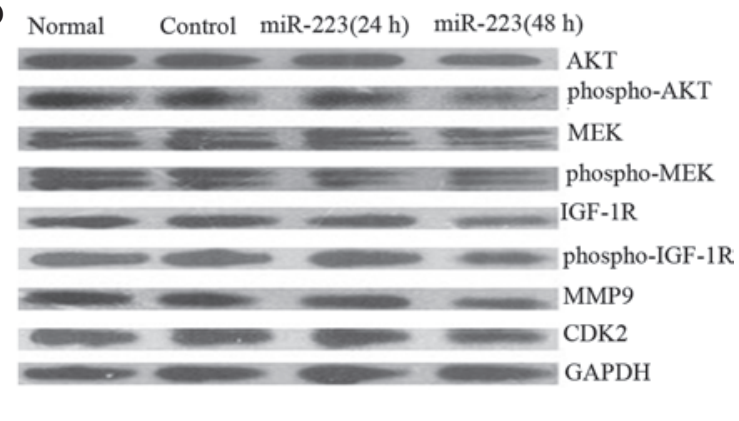

Figure 5. miR-223 regulates the activity of the IGF-1R signaling pathway and the expression of its key genes in LLC cells. (A) Sequence alignment of the miR-223 seed region and mRNA targets. (B) Luciferase 3'-UTR reporter assays of miR-223-induced gene silencing effects. (C) Expression of IGF-1R, CDK2 and MMP9 mRNA was significantly suppressed by transfection of cells with pre-miR-223, as confirmed by quantitative (q)PCR in LLC. (D) Detection and comparison of IGF-1R, CDK2, MMP9 and multiple phosphorylated kinase expression was altered following transfection with miR-223 or control vector by western blot analysis. GAPDH was used as loading control. ${ }^{*} \mathrm{P}<0.05,{ }^{* *} \mathrm{P}<0.01 \mathrm{vs}$. control. All data are representative of three independent experiments. miR, microRNA; LLC, Lewis lung carcinoma; UTR, untranslated region.

by miR-223, the expression of IGF-1R, Akt, ERK and their active forms (p-IGF-1R, p-Akt and p-ERK) was measured. The expression of IGF-1R, p-IGF-1R, p-Akt and p-ERK was reduced, however, the total Akt and ERK levels were unaffected (Fig. 5D). The downregulation of MMP9 expression at the mRNA and protein levels was further supported by qPCR and western blot analysis (Fig. 5C and D). These results indicate that the IGF-1R-mediated downstream signaling pathway was also affected by miR-223.

\section{Discussion}

The current study is the first study to demonstrate that miR-223 may be involved in lung cancer stem cell self-renewal and to show that miR-223 functions as a tumor suppressor in lung cancer cells at multiple steps of tumorigenesis and progression. In addition, IGF-1R and CDK2 were demonstrated to represent two significant targets for miR-223, crucial for mediating the miR-223-regulated malignant phenotype of LLC cells.

In the present study, IGF-1R expression and its phosphorylation levels in LLC were investigated and found to be markedly reduced, while cell proliferation was inhibited, following miR-223 overexpression. In addition, analyses using a IGF-1R 3'UTR reporter revealed a significant decrease in luciferase activity. In conclusion, these observations demonstrate that IGF-1R is the functional target of miR-223. IGF-1R is a transmembrane receptor tyrosine kinase encoded by a gene located on chromosome $15 q 26.3$. IGF-1R is implicated in the promotion of oncogenic transformation, growth and the survival of cancer cells $(24,25)$. It has been previously reported that IGF signaling mediates the transformation of normal lung cells and is involved in tumor initiation $(26,27)$. In addition, a number of studies in human lung cancer cell lines have demonstrated that the downregulation of IGF-IR inhibits lung tumor cell proliferation and sensitizes lung cancer cells to chemotherapy and radiotherapy $(28,29)$.

miR-223 has also been found to also target the CDK2 3'UTR. CDK2 is an S-phase cyclin-dependent kinase that is required for $\mathrm{p} 53$-independent $\mathrm{G}_{2} / \mathrm{M}$ checkpoint control. The inhibition of cyclin $\mathrm{A} / \mathrm{cdk} 2$ activation contributes to the maintenance of $\mathrm{G}_{2}$ phase arrest in response to DNA damage $(30,31)$. In human leukemia cells, inhibitors of ERK have been reported to increase the phosphorylation of cdc 25 c expression at the $\mathrm{G}_{2} / \mathrm{M}$ arrest stages and decrease $\mathrm{p} 21$ and CDK2 expression at the endoreduplication stages (22). Previous studies have demonstrated that the cyclolignan, picropodophyllin, downregulates IGF-1R tyrosine kinase activity and induces a marked accumulation of cells in the $\mathrm{G}_{2} / \mathrm{M}$-phase, as well as increased apoptosis (32). These observations, together with the results of the present study, are consistent with a model in which miR-223 induces $\mathrm{G}_{2} / \mathrm{M}$ arrest via the downregulation of IGF-1R and CDK2 by co-targeting their 3'UTR regions.

The present study primarily focused on whether the IGF-1R-mediated downstream signaling pathway is affected by $\mathrm{miR}-223$. The ERK and PI3K/Akt signaling pathways are central to the regulation of MMP9 expression $(33,34)$. The present results indicated that miR-223-decreased MMP9 activity was mediated by the suppression of phospho-ERK1/2 or phospho-Akt. In addition, the forced expression of miR-223 was found to downregulate Sca-1 in the LLC cells, as well as a simultaneous decrease of the cells in the $G_{0} / G_{1}$ phase and the induction of the inhibition of anchorage-independent 
growth. Sca-1, or Ly6A, is a member of the Ly6 family of glycosyl phostidylinositol-anchored cell surface proteins that is associated with murine stem/progenitor cells $(35,36)$. The MAPK/ERK pathway is essential for Sca- $1^{+}$hepatic progenitor cell proliferation and colony formation (37). In addition, IGF-1 stimulation directly induces anoikis resistance of a number of varying epithelial cell types by activating downstream signaling molecules, including Ras/MAPK and PI3K/Akt (38). In a colon cancer model, the kinase activities of Akt and ERK1/2 were shown to be significantly upregulated in $\mathrm{CD}_{133}{ }^{+}$cells (39). The clonogenic growth of the $\mathrm{CD}_{133^{+}}$cells was reduced markedly by inhibiting the activity of AKT and ERK1/2. In agreement with these results, we hypothesize that miR-223 induces an aberrant self-renewal capacity in LLC cells, at least in part, via the inhibition of AKT and ERK activity. Future studies must be performed to verify this hypothesis and identify the underlying mechanisms.

In the current study, miR-223 was revealed to function as a tumor suppressor in lung cancer. The results also indicate that miR-223 may fine-tune the activity of the IGF-1R pathway. These observations may provide a basis for novel therapies targeting IGF-1R in the treatment of NSCLC.

\section{Acknowledgements}

The present study was supported, in part, by grants from the National Natural Science Foundation of China (no. 30901790) and the Chongqing Natural Science Foundation (no. 2008BB5117).

\section{References}

1. Jemal A, Center MM, DeSantis C and Ward EM: Global patterns of cancer incidence and mortality rates and trends. Cancer Epidemiol Biomarkers Prev 19: 1893-1907, 2010.

2. Jemal A, Bray F, Center MM, Ferlay J, Ward E and Forman D: Global cancer statistics. CA Cancer J Clin 61: 69-90, 2011.

3. Bartel DP: MicroRNAs: genomics, biogenesis, mechanism and function. Cell 116: 281-297, 2004

4. Bueno MJ, Pérez de Castro I and Malumbres M: Control of cell proliferation pathways by microRNAs. Cell Cycle 7: 3143-3148, 2008.

5. Lee CT, Risom T and Strauss WM: MicroRNAs in mammalian development. Birth Defects Res C Embryo Today 78: 129-139, 2006.

6. Jovanovic $M$ and Hengartner MO: miRNAs and apoptosis: RNAs to die for. Oncogene 25: 6176-6187, 2006.

7. Mendell JT: miRiad roles for the miR-17-92 cluster in development and disease. Cell 133: 217-222, 2008.

8. Esquela-Kerscher A and Slack FJ: Oncomirs - microRNAs with a role in cancer. Nat Rev Cancer 6: 259-269, 2006.

9. Kent OA and Mendell JT: A small piece in the cancer puzzle: microRNAs as tumor suppressors and oncogenes. Oncogene 25 : 6188-6196, 2006.

10. Kumar MS, Erkeland SJ and Pester RE: Suppression of non-small cell lung tumor development by the let-7 microRNA family. Proc Natl Acad Sci USA 105: 3903-3908, 2008.

11. Fazi F, Rosa A, Fatica A, Gelmetti V, De Marchis ML, Nervi C and Bozzoni I: Aminicircuitry comprised of microRNA-223 and transcription factors NFI-A and C/EBPalpha regulates human granulopoiesis. Cell 123: 819-831, 2005.

12. Johnnidis JB, Harris MH, Wheeler RT, Stehling-Sun S, Lam MH and Kirak O: Regulation of progenitor cell proliferation and granulocyte function by microRNA-223. Nature 451: 1125-1129, 2008.

13. Jia CY, Li HH, Zhu XC, Dong YW, Fu D, Zhao QL, Wu W and Wu XZ: MiR-223 suppresses cell proliferation by targeting IGF-1R. Plos One 6: e27008, 2011.
14. Liu Q, Zhang M, Jiang X, Zhang Z, Dai L, Min S, Wu X, He Q, Liu J, Zhang Y, Zhang Z and Yang R: miR-223 suppresses differentiation of tumor-induced $\mathrm{CD}_{11 b^{+}} \mathrm{Grl}^{+}$myeloid-derived suppressor cells from bone marrow cells. Int J Cancer 129: 2662-2673, 2011.

15. Kang W, Tong JH, Chan AW, Lung RW, Chau SL, Wong QW, Wong N, Yu J, Cheng AS and To KF: Stathmin1 plays oncogenic role and is a target of microRNA-223 in gastric cancer. PLoS One 7: e33919, 2012

16. Li S, Li Z, Guo F, Qin X, Liu B, Lei Z, Song Z, Sun L, Zhang HT, You $\mathrm{J}$ and Zhou Q: miR-223 regulates migration and invasion by targeting Artemin in human esophageal carcinoma. J Biomed Sci 18: 24, 2011.

17. Wu L, Li H, Jia CY, Cheng W, Yu M, Peng M, Zhu Y, Zhao Q, Dong YW, Shao K, Wu A and Wu XZ: MicroRNA-223 regulates FOXO1 expression and cell proliferation. FEBS Lett 586: 1038-1043, 2012.

18. Izzotti A, Calin GA, Arrigo P, Steele VE, Croce CM and De Flora S: Downregulation of microRNA expression in the lungs of rats exposed to cigarette smoke. FASEB J 23: 806-812, 2009.

19. Heegaard NH, Schetter AJ, Welsh JA, Yoneda M, Bowman ED and Harris CC: Circulating micro-RNA expression profiles in early stage nonsmall cell lung cancer. Int J Cancer 130: 1378-1386, 2012.

20. Nian WQ, Chen FL, Ao XJ and Chen ZT: CXCR4 positive cells from Lewis lung carcinoma cell line have cancer metastatic stem cell characteristics. Mol Cell Biochem 355: 241-248, 2011.

21. Nian WQ, Chen FL, Ao XJ and Chen ZT: Lowly expression of miR-223 in CXCR4 positive cells from Lewis lung carcinoma cell line and its target gene prediction. Di San Jun Yi Da Xue Xue Bao 31: 2202-2205, 2009 (In Chinese).

22. Moon DO, Kim MO, Kang SH, Lee KJ, Heo MS, Choi KS, Choi $\mathrm{YH}$ and Kim GY: Induction of $\mathrm{G}_{2} / \mathrm{M}$ arrest, endoreduplication and apoptosis by actin depolymerization agent pextenotoxin-2 in human leukemia cells, involving activation of ERK and JNK. Biochem Pharmacol 76: 312-321, 2008.

23. Dufourny B, Alblas J and van Teeffelen HA: Mitogenic signaling of insulin-like growth factor I in MCF-7 human breast cancer cells requires phosphatidylinositol 3-kinase and is independent of mitogen-activated protein kinase. J Biol Chem 272: 31163-31171, 1997.

24. Khandwala HM, McCutcheon IE and Flyvbjerg A: The effects of insulin-like growth factors on tumorigenesis and neoplastic growth. Endocr Rev 21: 215-244, 2000.

25. Blakesley VA, Stannard BS and Kalebic T: Role of the IGF-I receptor in mutagenesis and tumor promotion. J Endocrinol 152: 339-344, 1997.

26. Moats-Staats BM, Price WA, Xu L, Jarvis HW and Stiles AD: Regulation of the insulin-like growth factor system during normal rat lung development. Am J Respir Cell Mol Biol 12: 56-64, 1995.

27. Linnerth NM, Siwicky MD, Campbell CI, Watson KL, Petrik JJ, Whitsett JA and Moorehead RA: Type I insulin-like growth factor receptor induces pulmonary tumorigenesis. Neoplasia 11: 672-682, 2009.

28. Goetsch L, Gonzalez A, Leger O, Beck A, Pauwels PJ, Haeuw JF and Corvaia N: A recombinant humanized anti-insulin-like growth factor receptor type I antibody (h7C10) enhances the antitumor activity of vinorelbine and anti-epidermal growth factor receptor therapy against human cancer xenografts. Int J Cancer 113: 316-328, 2005.

29. Cosaceanu D, Carapancea M, Castro J, Ekedahl J, Kanter L, Lewensohn R and Dricu A: Modulation of response to radiation of human lung cancer cells following insulin-like growth factor 1 receptor inactivation. Cancer Lett 222: 173-181, 2005.

30. Chung JH and Bunz F: Cdk2 Is required for p53-independent $\mathrm{G}_{2} / \mathrm{M}$ checkpoint control. PLoS Genet 6: e1000863, 2010.

31. Goldstone S, Pavey S, Forrest A, Sinnamon J and Gabrielli B: Cdc25-dependent activation of cyclin A/cdk2 is blocked in $\mathrm{G}_{2}$ phase arrested cells independently of ATM/ATR. Oncogene 209: 921-932, 2001.

32. Strömberg T, Ekman S, Girnita L, Dimberg LY, Larsson O, Axelson M, Lennartsson J, Hellman U, Carlson K, Osterborg A, Vanderkerken K, Nilsson K and Jernberg-Wiklund H: IGF-1 receptor tyrosine kinase inhibition by the cyclolignan PPP induces $\mathrm{G}_{2} / \mathrm{M}$-phase accumulation and apoptosis in multiple myeloma cells. Blood 107: 669-678, 2006.

33. Lin CC, Kuo CT, Cheng CY, Wu CY, Lee CW, Hsieh HL, Lee IT and Yang CM: IL-1 $\beta$ promotes A549 cell migration via MAPKs/AP-1- and NF- $\mathrm{BB}$-dependent matrix metalloproteinase-9 expression. Cell Signal 21: 1652-1662, 2009. 
34. Ellerbroek SM, Halbleib JM, Benavidez M, Warmka JK, Wattenberg EV, Stack MS and Hudson LG: Phosphatidylinositol 3-kinase activity in epidermal growth factor-stimulated matrix metalloproteinase-9 production and cell surface association. Cancer Res 61: 1855-1861, 2001.

35. Wu X, Pang L, Lei W, Lu W, Li J, Li Z, Frassica FJ, Chen X, Wan M and Cao X: Inhibition of Sca-1-positive skeletal stem cell recruitment by alendronate blunts the anabolic effects of parathyroid hormone on bone remodeling. Cell Stem Cell 7: 571-580, 2010.

36. Lu G, Haider HK, Jiang S and Ashraf M: Sca- $1^{+}$stem cell survival and engraftment in the infarcted heart: dual role for preconditioning induced connexin-43. Circulation 119: 2587-2596, 2009.
37. Jin C, Samuelson L, Cui CB, Sun Y and Gerber DA: MAPK/ERK and $\mathrm{Wnt} / \beta$-catenin pathways are synergistically involved in proliferation of Sca-1 positive hepatic progenitor cells. Biochem Biophys Res Commun 409: 803-807, 2011.

38. Valentinis B, Morrione A, Peruzzi F, Prisco M and Reiss K: Antiapoptotic signaling of the IGF-I receptor in fibroblasts following loss of matrix adhesion. Oncogene 18: 1827-1836, 1999.

39. Wang YK, Zhu YL, Qiu FM, Zhang T, Chen ZG, Zheng S and Huang J: Activation of Akt and MAPK pathways enhances the tumorigenicity of $\mathrm{CD} 133^{+}$primary colon cancer cells. Carcinogenesis 31: 1376-1380, 2010. 